\title{
A NEW GRADUATION ALGORITHM FOR COLOR BALANCE OF REMOTE SENSING IMAGE
}

\author{
Guoqing zhou, Xiaofan Liu, Tao Yue*, Qingyang Wang, Hongjun Sha, Shengxin Huang, Qiuyu Pan
}

Guangxi Key Laboratory of Spatial Information and Geomatics, Guilin University of Technology, No. 12 Jian'gan Road, Guilin, Guangxi 541004, China - (gzhou, yuetao)@ glut.edu.cn

\section{Commission III, WG III/IVb}

KEY WORDS: Remote sensing image, Mosaicking images, Gap line, Smooth transition, Color balance

\begin{abstract}
:
In order to expand the field of view to obtain more data and information when doing research on remote sensing image, workers always need to mosaicking images together. However, the image after mosaic always has the large color differences and produces the gap line. This paper based on the graduation algorithm of tarigonometric function proposed a new algorithm of Two Quarter-rounds Curves (TQC). The paper uses the Gaussian filter to solve the program about the image color noise and the gap line. The paper used one of Greenland compiled data acquired in 1963 from Declassified Intelligence Photography Project (DISP) by ARGON KH-5 satellite, and used the photography of North Gulf, China, by Landsat satellite to experiment. The experimental results show that the proposed method has improved the accuracy of the results in two parts: on the one hand, for the large color differences remote sensing image will become more balanced. On the other hands, the remote sensing image will achieve more smooth transition.
\end{abstract}

\section{INTRODUCTION}

Photography is the most intuitive access to obtain these data and information. We can obtain high-resolution satellite images and mapping products such as digital terrain models (DEM) with the technology improvement of remote sensing $(\mathrm{Li}$, 2002). It is necessary to connect many remote sensing images to obtain more data and information due to the limitation in single image. However, the photos will be affected by different seasons or other factors in the process of photos acquisition. As a result, mosaicking images will have obviously differences in brightness and produce a mosaic line. Therefore, it is necessary to propose an algorithm to make the image have smooth transition and color balance for the process of mosaicking remote sensing image.

At present, there are many methods have been proposed to eliminate the mosaicking line and smooth the image. On the one hand, using Histogram Specification, Histogram Equalization (Sun, 2015) or Fast Automatic Color Equalization (FACE) algorithm (Wang, 2009) before mosaicking image. On the other hands, using the algorithm of Wavelet, algorithm of Laplacian Pyramid Transform or others to mosaicking image (Gao, 2001). However, due to the limitation of the algorithm about FACE and Histogram Equalization, is difficulty to achieve image smooth. Although, algorithm of wavelet and algorithm of Laplacian Pyramid transform have good results but they have some disadvantages like great amount calculations, lack flexibility and they do not take into account the particularity of the image (Zhou, 2005). The main problem is that these algorithms can only achieve the noise reduction in the same direction, which can only meet darken or brighten at the same time, but cannot meet the image color balance with large color differences.Above of all disadvantages, Luo proposed the graduation algorithm of trigonometric function for images mosaic (Luo, 2004), which based on the graduation algorithm of Beinstein Polynomials. Zhou also proposed radiometric balancing (Zhou, 2002). The algorithm has some characteristics like fewer calculation and taken into account the factor of images particularity. But, the algorithm proposed by Luo also has some disadvantages, like the results has less colour orders and dose not suitable for long image mosaic.

Regarding the issues above all, the paper gives a graduation algorithm of Two Quarter-rounds Curves (TQC) . It can not only adapt the particularity of the image and settle the problem of large colour differences, but also achieve the noise reduction in the different direction. It has less calculations and more applicability. Then, using the Gaussian filter to smooth the mosaic line (Ma, 2017). Next, the paper will elaborate the principles of the new algorithm and using the remote sensing image of Greenland compiled data acquired in 1963 and the data of North gulf by Landsat satellite to experiment. At the end of the essay, the paper will give some advice and prospects.

\section{A NEW GRADUATION ALGORITHM}

\subsection{The Graduation Algorithm of Beinstein Polynomials}

2.1.1. Beinstein Polynomials: Beinstein polynomials is the polynomial of continuous and approximation which was named after Bernsteinov Bernstein, one of a famous mathematician in the field of numerical analysis. First, set function:

$$
\begin{gathered}
f:[0,1] \rightarrow R, \text { for } n \in N^{+} \\
B_{n}(f, x)=B_{n}(f)=\sum_{k=0}^{n} f\left(\frac{n}{k}\right) C_{n}^{k} x^{k}(1-x)^{n-k}
\end{gathered}
$$

For $x \in[0,1]$. Call $B_{n}(f, x)$ is the $\mathrm{n}$ order beinstein polynomials for function $\mathrm{f}$ (the order should no more than $\mathrm{n}$ ).

\footnotetext{
* Corresponding author: Tao Yue; E-mail: yuetao@glut.edu.cn.
} 
If $f(k \in n)$ is continuous at $[0,1]$, such that the derivative of order $\mathrm{k}$ is astringent to $\mathrm{f}$. If the $\mathrm{f}$ is discontinuity point of the first kind at $x_{0} \in(0,1)$, such that:

$$
B_{n}\left(f, x_{0}\right) \rightarrow \frac{f\left(x_{0}^{-}\right)+f\left(x_{0}^{+}\right)}{2}
$$

For $n \in \infty$. Above of all, the function was known as Beinstein Polynomials.

2.1.2. Graduation Algorithm of Beinstein Polynomials: Set image 3 is the image after mosaicking, image 1 and image 2 are two images apply to mosaicking, and have the formula such that:

$$
\text { image } 3=P * \text { image } 1+(1-p) * \text { image } 2
$$

For the $\mathrm{p}$ is gradual coefficient. We were known as Graduation Algorithm. And then, consider about the Beinstein Polynomials and set $p=\frac{n}{\text { width }}$, as shown in the figure 1 :

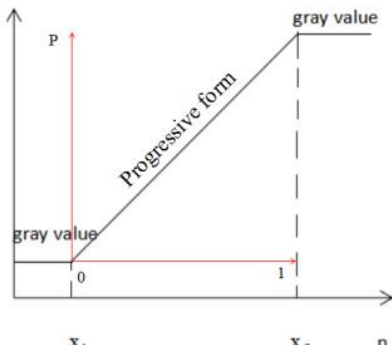

Figure.1 schematic of Beinstein Polynomials graduation algorithm

For the width is the image length, the $\mathrm{n}$ represent the image rows and must be aware that $n \leq w i d t h$. We were known it as Graduation Algorithm of Beinstein Polynomials; we also named it classic algorithm.

\subsection{Graduation Algorithm of Trigonometric Function}

The Graduation Algorithm of Trigonometric Function proposed by Luo was basis of The Graduation Algorithm of Beinstein Polynomials (Luo, 2002).

In classic algorithm, Luo found the algorithm has two discontinuity point of the second kinds at nodes which may be produces a dramatic changes in brightness. Therefore, Luo used the gradual coefficient to make the two nodes slopes are 0 (Luo,2002). As shown in the figure 2:

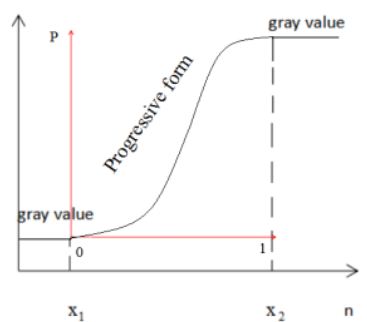

Figure. 2 schematic of trigonometric functin graduation algorithm
For the $\mathrm{n}$ represent images rows. As we know, the formula of sinusoidal function is:

$$
p=A \sin (\omega x+\varphi)+T
$$

Shift the origin to the left node. Set the left node to $x_{1}$. Similar, set the right node to $x_{2}$. Will $x_{1}$ and $x_{2}$ are stretched to the interval $[0,1]$. Such that:

$$
x^{\prime}=\frac{\left(x-x_{1}\right)}{\left(x_{2}-x_{1}\right)}
$$

Available

$$
p^{\prime}=0.5 \sin \left(\pi x^{\prime}-\frac{\pi}{2}\right)+0.5
$$

Substituting p' into the equation (3) and the result is the algorithm of trigonometric function.

\subsection{New Graduation Algorithm of TQC}

The experiments shows that two algorithms mentioned above have some disadvantages. On the one hand, algorithm of Bernstein Polynomial is the most simpleness but the gradual situation was hard and is not adapt to long image mosaic because of the less orders and gray scale mutations. On the other hands, although graduation algorithm of trigonometric function settled the problem of gray scale mutations, but it can not get a good visual effect due to the images are connected too smooth at the nodes when images have large colour differences before mosaicking image. In response to these problems, the paper proposed graduation algorithm of TQC.

2.3.1 Algorithm of TQC: The Q in TQC means quarter and it represents the quarter-round. it means using the curve for gradual coefficients by double quarter-rounds. The benefit of doing this can not only take into account the smooth and gradual of long image mosaic, but also consider the order rise sharply when images have large colour differences. As shown in the figure 3:

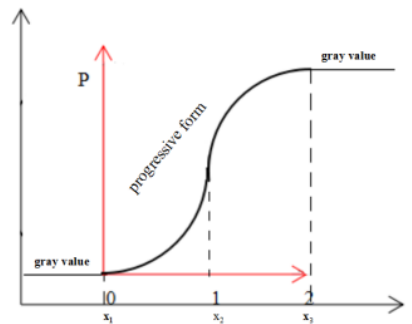

Figure. 3 schematic of TQC algorithm

First listed curve formula according to the circular curve formula:

$$
(x-a)^{2}+(x-b)^{2}=r^{2}
$$

For $x \in[0, r]$. Set the left node to $x_{1}$, set the position of mosaic line to $x_{2}$ and set right node to $x_{3}$. Will $x_{1}$ to $x_{2}$ are 
stretched to the interval $[0,1]$ and $x_{2}$ to $x_{3}$ are stretched to the interval [1,2] Such that:

$$
\left\{\begin{array}{l}
y_{1}=\sqrt{1-x_{1}^{2}}, x_{1} \in(0,1) \\
y_{2}=-\sqrt{1-\left(x_{2}-1\right)^{2}}+2, x_{2} \in(1,2)
\end{array}\right.
$$

The graduation coefficient is

$$
\left\{\begin{array}{l}
p=\sqrt{1-x_{1}^{2}}, x_{1} \in(0,1) \\
k=-\sqrt{1-\left(x_{2}-1\right)^{2}}+2, x_{2} \in(1,2)
\end{array}\right.
$$

As shown in the figure 3 , the select of graduation coefficient make the darker one brightening and make the brighter one darkening. This method not only improved the image colour balance, but also as the arc line gentle raise the graduation coefficient will have more colour orders to make image smooth and more adaptable to mosaicking image with long width.

2.3.2 Improve of Graduation Algorithm: The Histogram Equalization and Fast Automatic Color Equalization achieve image color balance by noise reduction in the same direction, as shown in the figure 4 :

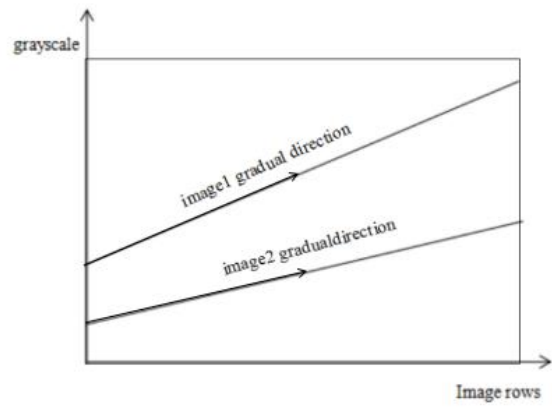

Figure.4 noise reduction in the same direction

These methods usually cannot achieve better color balance in the large color differences image.

Traditional graduation algorithm just consider the $\mathrm{p}$ is the coefficient to make image brighten, and then the $\mathrm{k}=1-\mathrm{p}$, so the $\mathrm{k}$ is the coefficient to make image darken. However, there is an important factor cannot be ignored that the color differences between image 1 to image2. It will not have a good visual effect by use $\mathrm{p}$ and $\mathrm{k}$ to make images smooth and gradual in this situation.

Above of all, I propose a new idea to improvement it. Studying this method, it is not difficult to find that both $\mathrm{p}$ and $\mathrm{k}$ are having symmetry. This paper make $\mathrm{p}$ and $\mathrm{k}$ are having asymmetry, so if the remote sensing image has large color differences, we can accord the specific situation to decide which part should change more intensely and obtain the more balanced color effect. At the same time, the algorithm can meet different direction noise reduction. As shown in the figure 5.

Set $p(n)$ is gradual function, for the $\mathrm{n}$ represent the rows of image ( $n \leq$ width). The $\mathrm{p}$ will from 0 to 1 as the $\mathrm{n}$ increase. The $\mathrm{k}$ will from 1 to2On the contrary, the $\mathrm{p}$ will from 1 to 0 . Such that:

$$
\text { image } 3=\left(1-p\left(n_{1}\right)\right) * \text { image } 1+p\left(n_{2}\right) * \text { image } 2
$$

$$
n_{1}=[1,2, \ldots, n-2, n-1, n], n_{2}=[1,2, \ldots, n-2, n-1, n]
$$

In this case, mosaicking image with large color difference will achieve smooth and gradual. At this moment, the graduation algorithm is:

$$
\text { image } 3=\text { image }^{*} p\left(n_{1}\right)+\text { image } 2 * k\left(n_{2}\right)
$$

$n_{1}=[1,2, \ldots, n-2, n-1, n], n_{2}=[1,2, \ldots, n-2, n-1, n]$

The above of the formula is known as The New Graduation Algorithm named TQC.

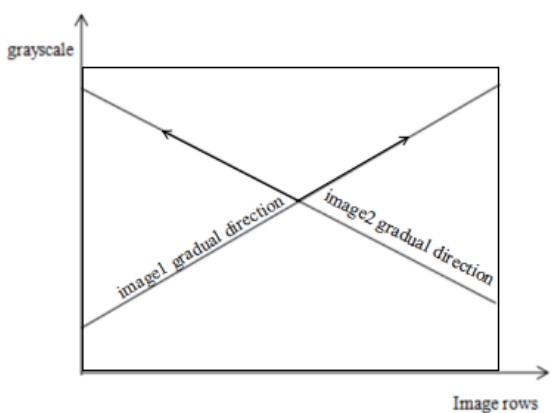

Figure.5 noise reduction in the different direction

\section{GAUSSIAN FILTER}

The Gauss filter is a kind of signal filter. In the field of digital image processing, it is always used to smooth the image. There are two ways to deal with the image, one is the discrete convolution and the other is the Fourier transform, we usually choose the first method to smooth the image. The Gauss filter is commonly used to deal with Gauss noise in the image i.e. the noise conforms to the Gauss distribution:

$$
G(x)=\frac{1}{\sqrt{2 \pi} \sigma} e^{-\frac{x^{2}}{2 \sigma^{2}}}
$$

The principle of Gaussian filter is building a mathematical model to convert the image data into energy. The part of image with low energy would be eliminated, and the image noise also belongs to the low energy part. The essence of gauss filter is choose a connected region and the pixel value of the intermediate point is equal to the mean of the pixel value of the all connected region, thus the image will achieving a smooth effect.

First, we should determine the pixel positions of the mosaic line and center on these pixel positions determine the field for Gaussian filter. Take the $3 \times 3$ pixel grids as an example, as shown in the figure 6 :

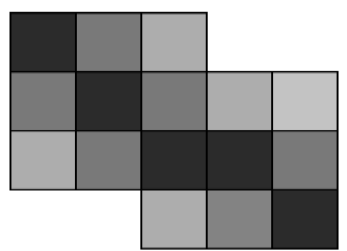

Figure. 6 the schematic of mosaic line, the black represent the pixel positions of the mosaic line 
Second, we should weight the mosaic line pixel values and multiply the relevant convolution kernels, output the result. As shown in the figure 7 :

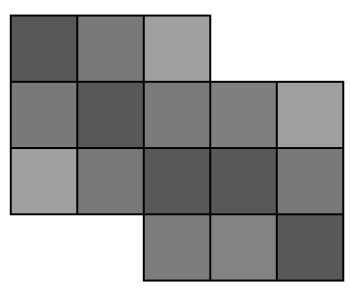

Figure.7 the pixels after Gaussian filter

As a result, the pixel values at the position of mosaic line and near it are weighted average. Visually, the image is smoother than before.

\section{EXPERIMENT AND SUMMARY}

\subsection{Comparison of Three Algorithms about Asymptotic Coefficients}

In order to verify the optimization effect of Quarter Algorithm, we will compare the value $\mathrm{P}$ (asymptotic coefficients) of the Bernstein Polynomial algorithm and the Trigonometric Function algorithm by experiments at the same time. At the end of experiments, compare some indexs include in the smoothness and the number of colour orders to test the algorithm is usefulness.

According to the principle of the Bernstein Polynomial algorithm, the Trigonometric Function algorithm and the TQC algorithm, we get the images of progressive effect. As shown in the figure 8 (1), (2), (3).
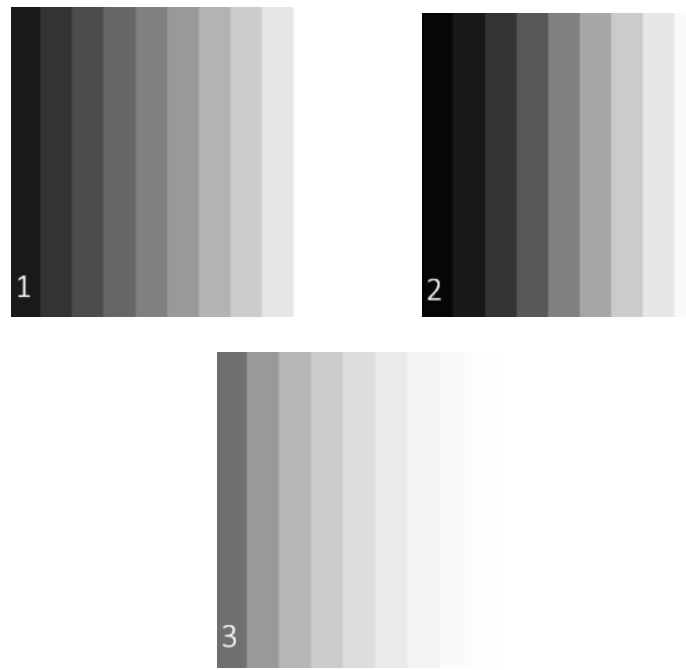

Figure. 8 compare of three algorithms about graduation coefficients that the (1) is the P of Bernstein Polynomial algorithm, the (2) is the $\mathrm{P}$ of Trigonometric Function algorithm and the (3) is the P of TQC algorithm

From the experiments we can see that the value $\mathrm{P}$ of Bernstein Polynomial algorithm was hard and had less colour orders, the images would not be smooth. The value $\mathrm{P}$ of Sinusoidal Function Algorithm also had less colour orders and it would not be suitable to long images mosaic.
To compare with algorithms above all, the value of the $\mathrm{P}$ in TQC has more orders and smooth. Therefore, it will have better effect in images mosaic then others.

\subsection{Application The New Graduation Algorithms of TQC}

Next, the paper wills application the technology to the images of reconnaissance satellite and Landsat satellite. First, we got the portion of remote sensing image in this paper. As shown in the figure 9:

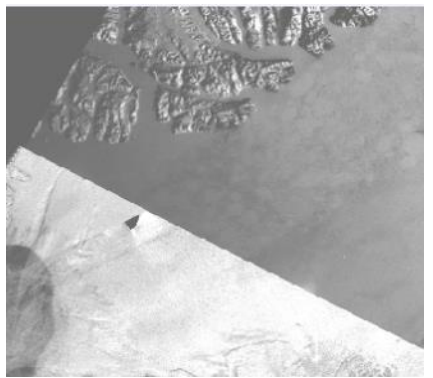

Figure. 9 the remote sensing image of Greenland acquired in 1963 by ARGON from reconnaissance satellite

Next, the paper will process this image by using the graduation algorithm of TQC, and treatment by Gaussian filter. As shown in the figure 10:

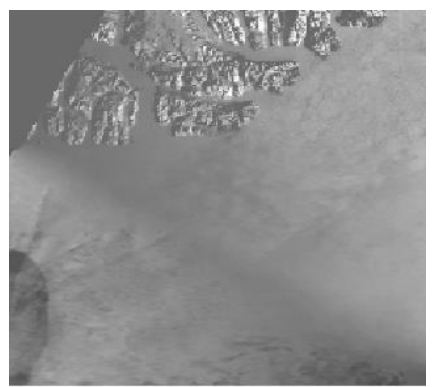

Figure.10 processed remote sensing image by the new graduation algorithm of Quarter

From the experiments, we can see that the graduation algorithm of TQC to deal with this image has better effect. Further, import the remote sensing image of North gulf, Guangxi, China, by Landsat satellite, which has different sensor as shown in the figure 11:

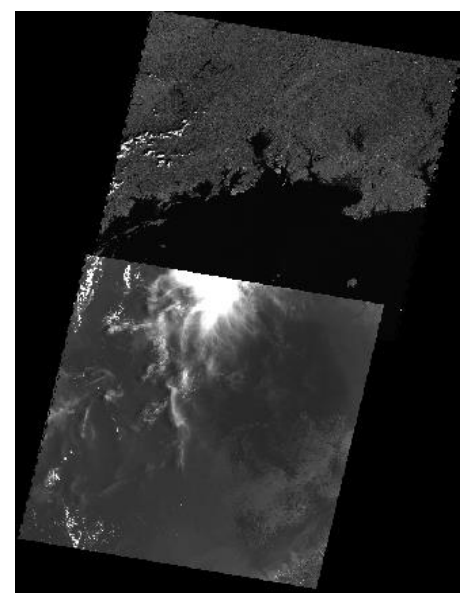

Figure.11 the image of North gulf, Guangxi, China 
The image has large color difference. Next, we will process this image by using TQC and treatment by Gaussian filter. As shown in the figure 12 :

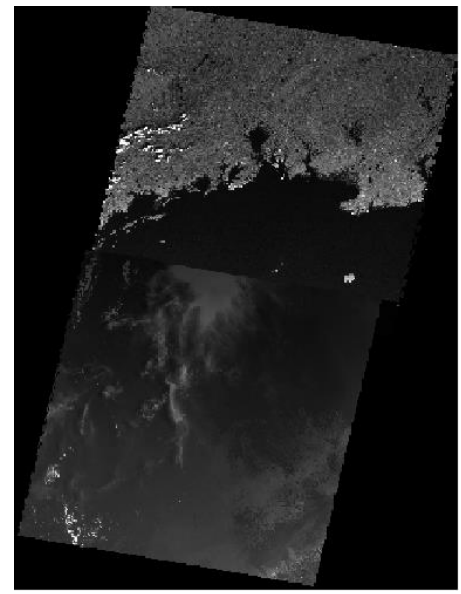

Figure.12 processed remote sensing image by the new graduation algorithm o TQC

Through experiments, we can draw such conclusion that using the new graduation algorithm of TQC to deal with remote sensing images will get a smooth and gradual result; the colour differences will decrease, and get a good result in the visual.

\section{CONCLUSION}

In this paper, we propose a new algorithm to smoothing the remote sensing image. The algorithm solves the problem with noise reduction in the different direction. Moreover, has advantages of less computational, more flexibility and adaptability to the particularity of image. It has a good visual effect by this algorithm to deal the images for the large difference, and it suitable for long-range remote sensing images mosaic. At the end of easy, the paper gave several groups of experimental images include the Greenland compiled data acquired in 1963 and the data from Landsat which had the particularity of itself like different mosaic line and difference of brightness and darkness. The experiment shows that the algorithm proposed by this paper is correct. It is not only can use for reconnaissance satellite but also can do it for Landsat satellite and so on. It can be used in more field. In the future, the algorithm, which can adapt to the particularity of the image will become the research hot spot.

\section{ACKNOWLEDGEMENTS}

This paper is financially supported by the National Natural Science of China under Grant numbers 41431179, the National Key Research and Development Program of China under Grant numbers 2016YFB0502501 and The State Oceanic Administration under Grant numbers 2014\#58, GuangXi Natural Science Foundation under grant numbers 2015GXNSFDA139032, and 2012GXNSFCB05300; Guangxi Science \& Technology Development Program under the Contract numbers GuiKeHe 141230014, GuangXi Key Laboratory of Spatial Information and Geomatics Program (Contract No.151400701, 151400712, and 163802512).

\section{REFERENCES}

Barsi, J. A., Schott, J. R., Palluconi, F. D., Helder, D. L., Hook, S. J. and Markham, B. L., et al., 2003a. Landsat tm and etm+ thermal band calibration. Canadian Journal of Remote Sensing, 29(2), pp.141-153.

Gao L. and Wei Q., 2001a. Study and Implementation of Image Merging Based on Wavelet Transform. Journal of Institute of Command and Technology,12(5), pp. 30-32.

Gu M., 2002a. Progress of the Solar Calibration Technique for Space-borne Multispectral Sensors. Chinese Space Science and Technology, 2, pp. 35-43.

Irons J. R., Dwyer J. L. and Barsi J. A., 2012a. The next Landsat satellite: The Landsat Data Continuity Mission. Remote Sensing of Environment, 122(4), pp. 11-21.

Li R., Zhou G., Schmidt N. J., Fowler C., Tuell G., 2002a. Photogrammetric Processing of High-resolution Airborne and Satellite Linear Array Stereo Images for Mapping Applications. International Journal of Remote Sensing, 23(20), pp.4451-4473.

Labandibar J. Y., Baudin G. and Ballion Y., 2004a. AlcatelSpace in hyper/multispectral Earth observation programs from Meris to Spectra. SPIE-The International Society for Optical Engineering, 5234, pp. 232-242.

Liberti L., Carilo A. and Sannino G. 2013a. Wave energy resource assessment in the Mediterranean, the Italian perspective. Renew Energy, 50 (9), pp.38-49.

Luo W., Li Y. and Wang X., 2004a. A New Graduation Algorithm in Image Mosaic. Computer Engineering and Applications, 36, pp. 50-54.

Markham B. L. and Helder D. L., 2012a. Forty-year calibration record of earth-reflected radiance from Landsat: A review. Remote Sensing of Environment, 122(3), pp. 30-40.

Ma L., Gao Y., Yin T. and Zhai W., 2017a. Improved Flight Conflict Detection Algorithm Based on Gauss-Hermite Particle filiter. WuHan University Journal of Natural Sciences, 22(3), pp. 269-276.

Wang Y. and Huang B., 2009a. Impoved Fast Automatic Colour Equalization Algorithm. Radio Communication Technology, 44(4), pp. 44-47.

Zhou G., 2009a. Near real-time orthorectificatoin and mapping of UAV-based video flow for time-critical event response, IEEE Transactions on Geoscience and Remote Sensing, 47(3), pp.739-747.

Zhou G., Chen W., Kelmelis J. and Zhang D., 2005a. A Comprehensive Study on Urban True Orthorectification. IEEE Transactions On Geoscience And Remote Sensing, 43(9), pp. $2138-2147$

Zhou G. and Jezek K. C., 2002a. Satellite photograph mosaics of Greenland from the 1960 s era. International Journal of Remote Sensing, 23(6), pp. 1143-1159. 